\title{
Connectivity and Dynamics Underlying Synaptic Control of the Subthalamic Nucleus
}

\author{
Leon Amadeus Steiner, ${ }^{1}$ Federico J. Barreda Tomás, ${ }^{2}$ Henrike Planert, ${ }^{1}$ Henrik Alle, ${ }^{1}$ Imre Vida, ${ }^{2,3}$ \\ and Jörg R.P. Geiger ${ }^{1,3}$ \\ ${ }^{1}$ Institute of Neurophysiology, Charité-Universitätsmedizin Berlin, 10117 Berlin, Germany, ${ }^{2}$ Institute of Integrative Neuroanatomy, \\ Charité-Universitätsmedizin Berlin, 10117 Berlin, Germany, and ${ }^{3}$ NeuroCure Cluster of Excellence, Charité-Universitätsmedizin Berlin, 10117 Berlin, \\ Germany
}

\begin{abstract}
Adaptive motor control critically depends on the interconnected nuclei of the basal ganglia in the CNS. A pivotal element of the basal ganglia is the subthalamic nucleus (STN), which serves as a therapeutic target for deep brain stimulation (DBS) in movement disorders, such as Parkinson's disease. The functional connectivity of the STN at the microcircuit level, however, still requires rigorous investigation. Here we combine multiple simultaneous whole-cell recordings with extracellular stimulation and post hoc neuroanatomical analysis to investigate intrinsic and afferent connectivity and synaptic properties of the STN in acute brain slices obtained from rats of both sexes. Our data reveal an absence of intrinsic connectivity and an afferent innervation with low divergence, suggesting that STN neurons operate as independent processing elements driven by upstream structures. Hence, synchrony in the STN, a hallmark of motor processing, exclusively depends on the interactions and dynamics of GABAergic and glutamatergic afferents. Importantly, these inputs are subject to differential short-term depression when stimulated at high, DBS-like frequencies, shifting the balance of excitation and inhibition toward inhibition. Thus, we present a mechanism for fast yet transient decoupling of the STN from synchronizing afferent control. Together, our study provides new insights into the microcircuit organization of the STN by identifying its neurons as parallel processing units and thus sets new constraints for future computational models of the basal ganglia. The observed differential short-term plasticity of afferent inputs further offers a basis to better understand and optimize DBS algorithms.
\end{abstract}

Key words: high-frequency stimulation; minimal stimulation; multipatch recordings; short-term plasticity; subthalamic nucleus; synaptic connectivity

\section{Significance Statement}

The subthalamic nucleus (STN) is a pivotal element of the basal ganglia and serves as target for deep brain stimulation, but information on the functional connectivity of its neurons is limited. To investigate the STN microcircuitry, we combined multiple simultaneous patch-clamp recordings and neuroanatomical analysis. Our results provide new insights into the synaptic organization of the STN identifying its neurons as parallel processing units and thus set new constraints for future computational models of the basal ganglia. We further find that synaptic dynamics of afferent inputs result in a rapid yet transient decoupling of the STN when stimulated at high frequencies. These results offer a better understanding of deep brain stimulation mechanisms, promoting the development of optimized algorithms.

\section{Introduction}

Adaptive motor control in vertebrates relies on the integrative properties of interconnected neuronal networks, including the

Received June 29, 2018; revised Dec. 29, 2018; accepted Jan. 24, 2019.

Author contributions: L.A.S., F.J.B.T., H.A., I.V., and J.R.P.G. designed research; L.A.S., F.J.B.T., and H.P. performed research; L.A.S., F.J.B.T., and H.P. analyzed data; L.A.S. and F.J.B.T. wrote the first draft of the paper; L.A.S., F.J.B.T., H.P., H.A., I.V., and J.R.P.G. edited and wrote the paper.

This work was supported by German Research Foundation Grant KF0 247, the Cluster of Excellence NeuroCure EXC 257, and the Research Training Group GRK 1589. We thank Yangfan Peng for the design of the multiple patch-clamp recording setup and initial training; Ina Wolter for technical support with the immunocytochemical and histological processing; and Andrew Sharott, Wolf-Julian Neumann and Michael Daniel Hadler for helpful comments on an earlier version of the man- motor cortex and the structures of the basal ganglia (StephensonJones et al., 2011). Among the latter, the subthalamic nucleus (STN) is the only glutamatergic nucleus (Bolam et al., 2000). Further, it occupies a pivotal position within these circuits: it integrates complex afferent input, most prominently the cortical

uscript. VGAT-Venus transgenic rats were generated by Drs. Y. Yanagawa, M. Hirabayashi, and Y. Kawaguchi (National Institute for Physiological Sciences, Okazaki, Japan), using p(S2-Venus provided by Dr. A. Miyawaki.

The authors declare no competing financial interests.

Correspondence should be addressed to Jörg R.P. Geiger at joerg.geiger@charite.de.

https://doi.org/10.1523/JNEUROSCI.1642-18.2019

Copyright $\odot 2019$ the authors $\quad 0270-6474 / 19 / 392470-12 \$ 15.00 / 0$ 
"hyperdirect" (Nambu et al., 2002) and the pallidal "indirect" pathway (Smith et al., 1990), and targets the main output nuclei of the basal ganglia. The STN has also received substantial attention as a major target for deep brain stimulation (DBS) to treat symptoms of Parkinson's disease (PD) (Benabid et al., 1994; Chen et al., 2006). Despite increasing interest in the physiology of the STN and its clinical relevance, however, intrinsic and afferent connectivity of STN neurons and their synaptic properties are not fully explored.

Under physiological conditions, neurons in the basal ganglia engage in brief transient synchronization at beta frequencies, and the dynamics of synchronization are instrumental to basal ganglia function (Feingold et al., 2015; Mirzaei et al., 2017; Tinkhauser et al., 2017a). Conversely, beta activity recorded in the STN is exaggerated in PD (Neumann et al., 2016; Steiner et al., 2017; Tinkhauser et al., 2017a), and clinically effective DBS reduces synchrony in this nucleus (Kühn et al., 2008). Nevertheless, little is known of the anatomical substrate underlying synchronization in the STN. As neuronal network synchrony may depend on both afferent and intrinsic connectivity, prime candidates, which could mediate synaptic control of neuronal synchrony in the STN, include the following: (1) glutamatergic afferents of the hyperdirect cortical input, (2) GABAergic afferents belonging to the indirect pathway, and (3) intranuclear mutual connectivity of STN neurons. In terms of afferent connectivity, there is limited information on the organization of incoming GABAergic inputs (Baufreton et al., 2009); however, functional connectivity of glutamatergic projections, potentially more critical to the control of synchrony in the STN (Gradinaru et al., 2009; Li et al., 2012; Sanders and Jaeger, 2016), is not well understood. More specifically, incoming fibers of both indirect (Baufreton et al., 2009) and hyperdirect pathways collateralize in the STN (Kita and Kita, 2012), and their divergence may serve as an anatomical prerequisite for synchronous recruitment. Mutual synaptic connectivity between STN neurons has been suggested on the basis of anatomical (Hammond and Yelnik, 1983; Kita et al., 1983; Chang et al., 1984; Ammari et al., 2010; Gouty-Colomer et al., 2018) and indirect electrophysiological observations (Shen and Johnson, 2006; Ammari et al., 2010; Chu et al., 2012), but these observations were called into question by contrasting findings (Wilson et al., 2004; Koshimizu et al., 2013). Thus, the mere existence of functional intranuclear connections remains contentious.

In the present study, we combine simultaneous whole-cell recordings of up to 7 neurons with extracellular stimulation and morphological analysis of the recorded neuronal clusters, to investigate the intrinsic and afferent functional connectivity of rat STN neurons. We further analyze functional properties and interactions of synaptic inputs to these neurons. Finally, we compare synaptic dynamics of both glutamatergic and GABAergic inputs in response to repetitive stimulation at low and high, DBS-like frequencies.

\section{Materials and Methods}

Slice preparation. Acute brain slices $(n=64)$ were prepared from 38 juvenile (P14-P21) transgenic Wistar rats of both sexes expressing Venus-YFP under the VGAT promoter (Uematsu et al., 2008) (RRID: RGD_2314361). To control for age-dependent effects, we performed additional experiments in a set of 4 adult animals (P61-P70). Animal handling and all procedures were performed in accordance with guidelines of local authorities (Berlin, [T0109/10]), the German Animal Welfare Act, and the European Council Directive 86/609/EEC. Animals were decapitated after receiving isoflurane anesthesia, and the head was immediately submerged in an ice-cold sucrose-based slicing solution containing the following (in mM): 196 sucrose, $2.5 \mathrm{KCl}, 1.2 \mathrm{NaH}_{2} \mathrm{PO}_{4}, 20$ glucose, $26 \mathrm{NaHCO}_{3}, 0.5 \mathrm{CaCl}_{2}, 3.5 \mathrm{MgCl}_{2}$ enriched with carbogen $(95 \%$ $\mathrm{O}_{2} / 5 \% \mathrm{CO}_{2}$ ).
Horizontal and parasagittal 300- $\mu$ m-thick slices containing the STN were cut using a VT1200 vibratome (Leica Microsystems). Acute slices of both horizontal and parasagittal orientation were cut to enable comparison of our results with those of previous studies working in either of these two planes. Subsequently, slices were stored in an ACSF containing the following (in mM): $126 \mathrm{NaCl}, 2.5 \mathrm{KCl}, 1.2 \mathrm{NaH}_{2} \mathrm{PO}_{4}, 11$ glucose, 19 $\mathrm{NaHCO}_{3}, 2.4 \mathrm{CaCl}_{2}, 1.2 \mathrm{MgCl}_{2}$, bubbled with carbogen $\left(95 \% \mathrm{O}_{2} / 5 \%\right.$ $\mathrm{CO}_{2}$ ). For recovery, slices were kept at $34^{\circ} \mathrm{C}$ for a minimum of $30 \mathrm{~min}$. Slices were stored in an interface-type chamber in carbogenated ACSF for up to $5 \mathrm{~h}$ before being transferred to the recording chamber.

Whole-cell patch-clamp recordings. Recordings were performed in a submerged-type recording chamber continuously perfused with ACSF held at $34^{\circ} \mathrm{C}$. Somatic whole-cell patch-clamp recordings were performed using pipettes pulled from borosilicate glass capillaries $(2 \mathrm{~mm}$ outer/1 mm inner diameter) on a horizontal puller (P-97, Sutter Instrument). The pipettes were filled with an intracellular solution containing the following (in mM): $145 \mathrm{~K}$-gluconate, $6 \mathrm{KCl}, 10 \mathrm{HEPES}, 0.2$ EGTA, 5 $\mathrm{Na}_{2}$-phosphocreatine, $2 \mathrm{Na}_{2} \mathrm{ATP}, 0.5 \mathrm{Na}_{2} \mathrm{GTP}$, and $2 \mathrm{MgCl}_{2}(290-300$ mOsm, $\mathrm{pH}$ adjusted to 7.2 with $\mathrm{KOH}$ ); $0.1 \%$ biocytin was added for morphological analysis in a subset of experiments. Filled pipettes had a resistance of 3-7 $\mathrm{M} \Omega$. Membrane potential values given in the text are not corrected for the liquid junction potential.

Cells were visualized using infrared differential interference contrast video microscopy (BX-51WI, Olympus). The STN was identified as an almond-shaped structure in close proximity to the internal capsule and substantia nigra pars reticulata (SNr; Fig. 1A). Identification was confirmed by probing VGAT-YFP fluorescence in epifluorescence illumination using a $490 \mathrm{~nm}$ LED light source (Thorlabs). The STN was homogeneously YFPnegative, in contrast to the neighboring SNr.

We recorded from up to 7 cells simultaneously in depths of up to $72 \mu \mathrm{m}$ beneath slice surface (mean \pm SEM: $39 \pm 1 \mu \mathrm{m}$; Fig. $1 B$ ). The series resistance in current-clamp recordings was compensated using the automated bridge balance compensation of the amplifier. Recordings were performed using 4 two-channel Multiclamp 700B amplifiers (Molecular Devices). Data were low-pass filtered at $6 \mathrm{kHz}$ using the amplifiers built-in Bessel filter and digitized with a Digidata 1550 (Molecular Devices) at a sampling rate of 20 $\mathrm{kHz}$. The pClamp 10.3.0.7 software package (Molecular Devices) was used for data acquisition and analysis. Recorded cells had a resting membrane potential of $-60 \pm 1 \mathrm{mV}$ (mean \pm SEM).

Synaptic connectivity screening. Trains of 4 action potentials (APs) at 20 $\mathrm{Hz}$, a physiological burst frequency in the STN (Tinkhauser et al., 2017a), were elicited in a single cell by injecting 1- to 2-ms-long suprathreshold current pulses of 1-2.5 nA. Each recording sweep was $8 \mathrm{~s}$ long, and the individual cells were stimulated sequentially in $1 \mathrm{~s}$ intervals; therefore, each cell was activated once every $8 \mathrm{~s}(0.125 \mathrm{~Hz})$. For the analysis of synaptic connectivity, 20-40 sweeps were averaged. All postsynaptic traces were thoroughly examined for postsynaptic potentials with a maximum latency of $<3$ ms to presynaptic APs, allowing for the detection of unitary postsynaptic potentials as small as $40 \mu \mathrm{V}$ in average amplitude (Böhm et al., 2015; Peng et al., 2017).

Visualization of recorded neurons. After recording and concomitantly filling the cells with biocytin, slices were immersion-fixed in a solution containing $4 \%$ PFA and $4 \%$ sucrose in $0.1 \mathrm{M} \mathrm{PB}$ for a minimum of $12 \mathrm{~h}$ (overnight) at $4^{\circ} \mathrm{C}$. Slices were then rinsed extensively in $0.1 \mathrm{M} \mathrm{PB}$ and subsequently permeabilized in a solution containing $0.3 \%-0.5 \%$ Triton $\mathrm{X}-100$ in $0.1 \mathrm{M}$ PB. Processed and biocytin-containing cells were visualized using avidin-conjugated AlexaFluor-647 (Thermo Fisher Scientific; dilution 1:500; RRID:AB_2336066) before being coverslipped using an aqueous mounting medium. Imaging of the slices was performed on a confocal laser-scanning microscope (Olympus FluoView FV1000) using a $4 \times$ objective for overview of cell clusters, and a $30 \times$ siliconeimmersion objective (numerical aperture, 1.05) to obtain image stacks for the assessment of single-cell morphology. Fluorescence emission from YFP-labeled putative GABAergic cells was elicited by the $480 \mathrm{~nm}$ line of an Argon laser. A $643 \mathrm{~nm}$ laser diode was used to visualize the AlexaFluor-647 in biocytin-labeled neurons (Fig. 1D). Selected cells were morphologically reconstructed using the Simple Neurite Tracer plug-in (Longair et al., 2011) in the Fiji distribution of ImageJ software (National Institutes of Health; RRID:SCR_003070; Fig. 1E). 
A

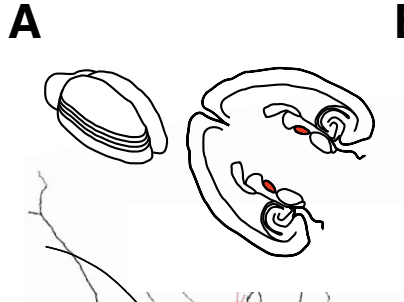

B
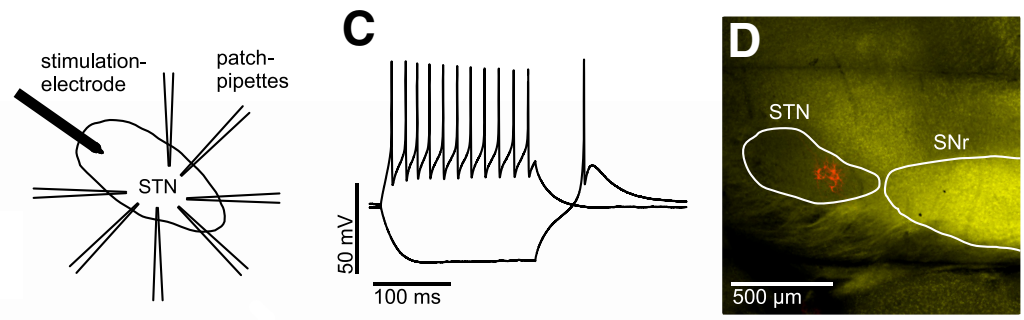

$\mathbf{E}$
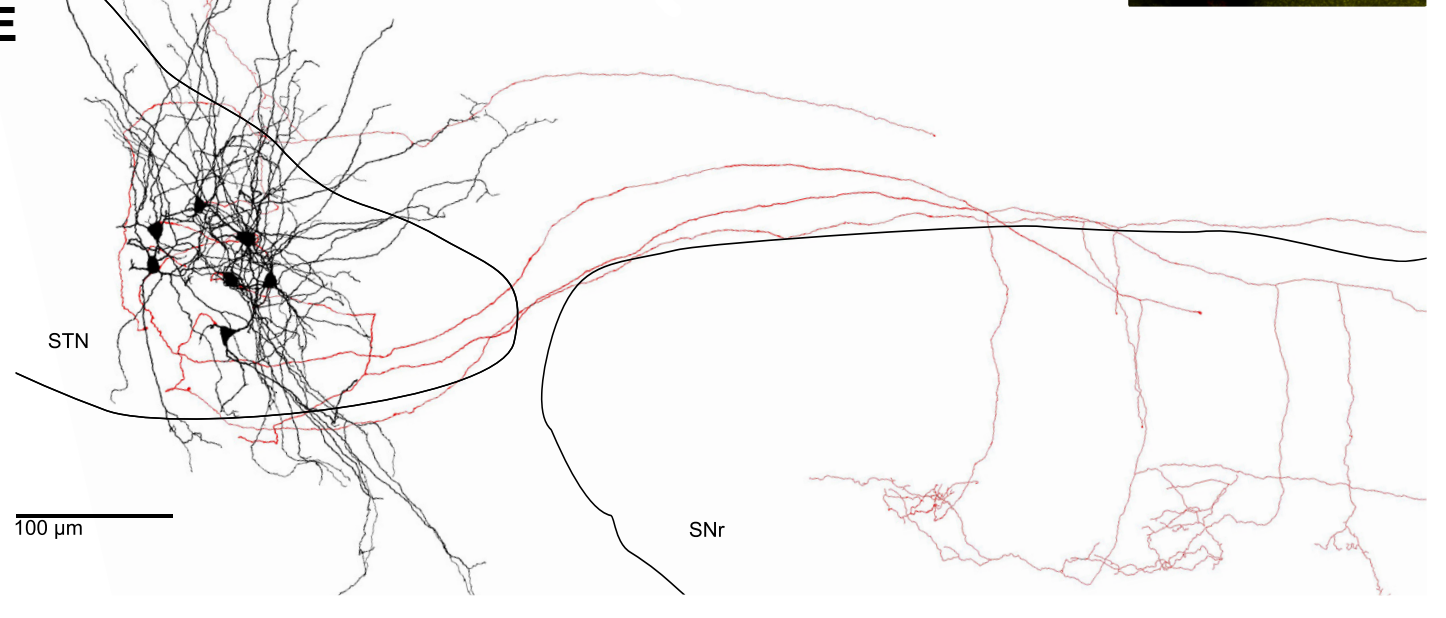

Figure 1. Electrophysiological and morphological characterization of clusters of neurons within the STN. $A$, Left, Schematic drawing of an acutely isolated rat brain. Parallel lines indicate orientation of subsequently obtained "horizontal" slices. Right, Anatomical landmarks in a horizontal brain slice with the STN in each hemisphere highlighted in red. $\boldsymbol{B}$, Experimental paradigm: up to 7 STN neurons were recorded simultaneously. An extracellular stimulation electrode was placed at the rostral tip of the STN to stimulate axons afferent to the cluster of recorded neurons. C, AP pattern of an STN neuron in response to a depolarizing current injection superimposed on a voltage trace in response to a hyperpolarizing current injection, which results in a rebound spike characteristic for STN neurons. D, Fluorescence microscopic image of a horizontal slice obtained from a VGAT-YFP rat (see Materials and Methods) containing both the STN (VGAT-YFP-negative) and the SNr (VGAT-YFP-positive). Red-labeled structures in the STN represent seven simultaneously recorded and biocytin-filled neurons. $\boldsymbol{E}$, Reconstruction of the same cluster of neurons shown in $\boldsymbol{D}$. Black represents dendrites and somata. Red represents axons. Note the partially preserved axonal projections to the SNr.

Analysis of spatial proximities between STN neurons. Spatial proximities between pairs of recorded STN neurons were screened for using the ImageJ software. We examined confocal image stacks of 5 cell clusters from 5 animals. Potential contacts were classified as contacts originating from main axon segments or axon collaterals. Reconstructed cell pairs from recorded cell clusters were analyzed for potential contacts using a custom NEURON (https://neuron.yale.edu/neuron/) script. Number and dendritic length of potential contacts within $4 \mu \mathrm{m}$ radius (center to center) from the central axis of the axon were determined per cell pair from the recorded clusters.

Placement of stimulation electrode/extracellular stimulation settings. For extracellular stimulation, a tungsten bipolar electrode (tip diameter $\sim 30$ $\mu \mathrm{m}$ ) was placed in the rostral part of the STN (Fig. 1B). The electrode was lowered $\sim 50 \mu \mathrm{m}$ into the slice. Stimulation intensity varied between 8 and $500 \mu \mathrm{A}$ across experiments, with a pulse duration of $100 \mu \mathrm{s}$.

Drugs. Drugs were purchased from Tocris Bioscience. Concentrated stock solutions of gabazine (GABA-A-receptor antagonist), D-AP5, and $\mathrm{CNQX}$ disodium salt (ionotropic glutamate-receptor antagonists) were made with $\mathrm{H}_{2} \mathrm{O}$ and diluted in ACSF immediately before use for bath application at final concentrations of 10,50 , and $10 \mu \mathrm{M}$, respectively.

Minimal stimulation paradigm. Stimulation intensity was increased in steps of $10 \mu \mathrm{A}$ until a postsynaptic response was seen in one of several simultaneously recorded cells, and subsequent stimulation intensity adjusted in steps of $1 \mu \mathrm{A}$ to precisely determine the minimal stimulation threshold. Divergence of afferents onto STN neurons within a recorded cluster was studied by assessing the parallel emergence of postsynaptic responses in simultaneously recorded cells. To study GABAergic transmission in more detail, glutamatergic transmission was blocked in a subset of experiments using D-AP5 and CNQX. Glutamatergic transmission was studied in isolation in the presence of gabazine.

Single-pulse stimulation. Single stimulation pulses of up to $500 \mu \mathrm{A}$ were applied without pharmacological manipulation to cause broad afferent activation and reveal the degree of overlay of GABAergic and glutamatergic synaptic responses within single cells and across a recorded cluster. Experiments were performed both in voltage- and current-clamp mode to study synaptic inputs and AP generation in STN neurons, respectively. In each experiment, we recorded 10 sweeps to screen signal variability. To disentangle AP generation in STN neurons from the influence of afferent GABAergic activity, experiments were performed in the presence of gabazine in a subset of experiments. While most experiments were performed at resting membrane potential, some cells were depolarized to -60 or $-50 \mathrm{mV}$ (in both the gabazine and nongabazine condition) to facilitate AP generation. Whenever single-pulse stimulation evoked APs in one of the recorded cells, we evaluated the first AP after stimulus. In a next step, we compared cell-specific median latencies and SDs of APs. SDs were omitted whenever less than two APs were recorded. On a group level, we compared between the gabazine and nongabazine condition across cells.

Variation of extracellular stimulation frequency. To study frequencydependent dynamics of synaptic inputs to STN neurons, stimuli of 500 $\mu \mathrm{A}$ were applied at 10,20, and $130 \mathrm{~Hz}$. Each stimulation train was applied for $1 \mathrm{~s}$, and the stimulation interval was followed by a $4 \mathrm{~s}$ break. Thus, the total sweep duration was $5 \mathrm{~s}$. A total of 10 sweeps were recorded for each stimulation frequency and averaged for subsequent analysis. Experiments with clear and reliable onset of synaptic responses (either compound glutamatergic or compound GABAergic) in all tested stimulation frequencies were included in further post hoc analysis. In a subset of experiments, it was necessary to extrapolate the stimulation artifact offset toward baseline to correctly assess EPSC or IPSC amplitude. To quantify and normalize synaptic depression for individual cells, we calculated synaptic depression ratios dividing the synaptic current amplitude evoked by the fifth stimulus by the synaptic current amplitude evoked by the first stimulus. To quantify synaptic recovery after DBS-like stimulation frequencies of 130 $\mathrm{Hz}$, we compared synaptic current amplitudes after the first stimulus in the first sweep to their counterparts in the 10th sweep. 
A

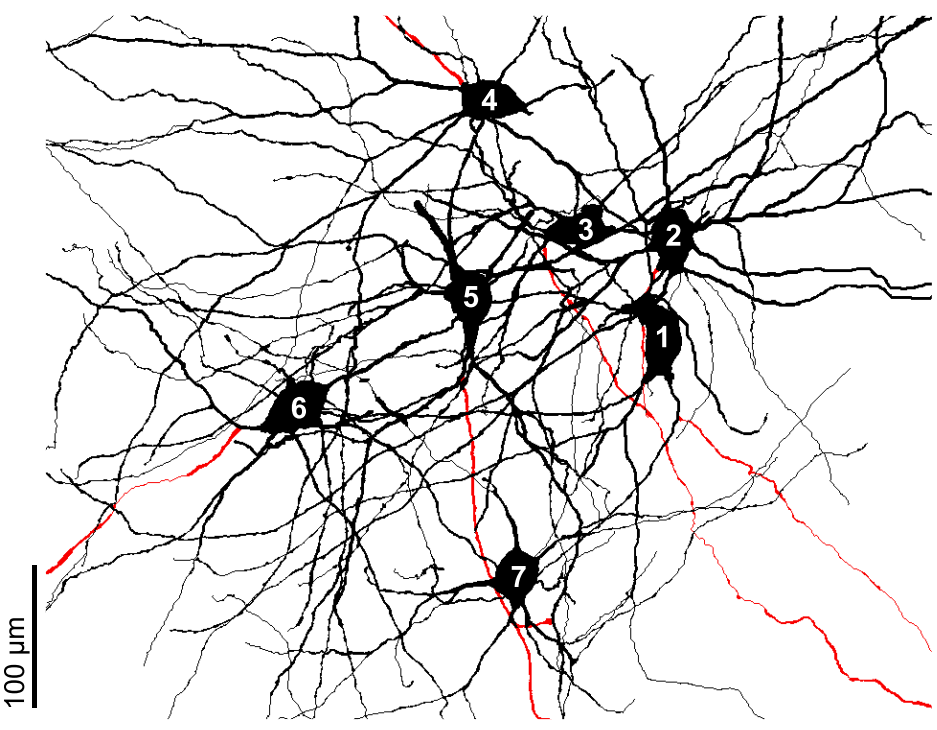

B

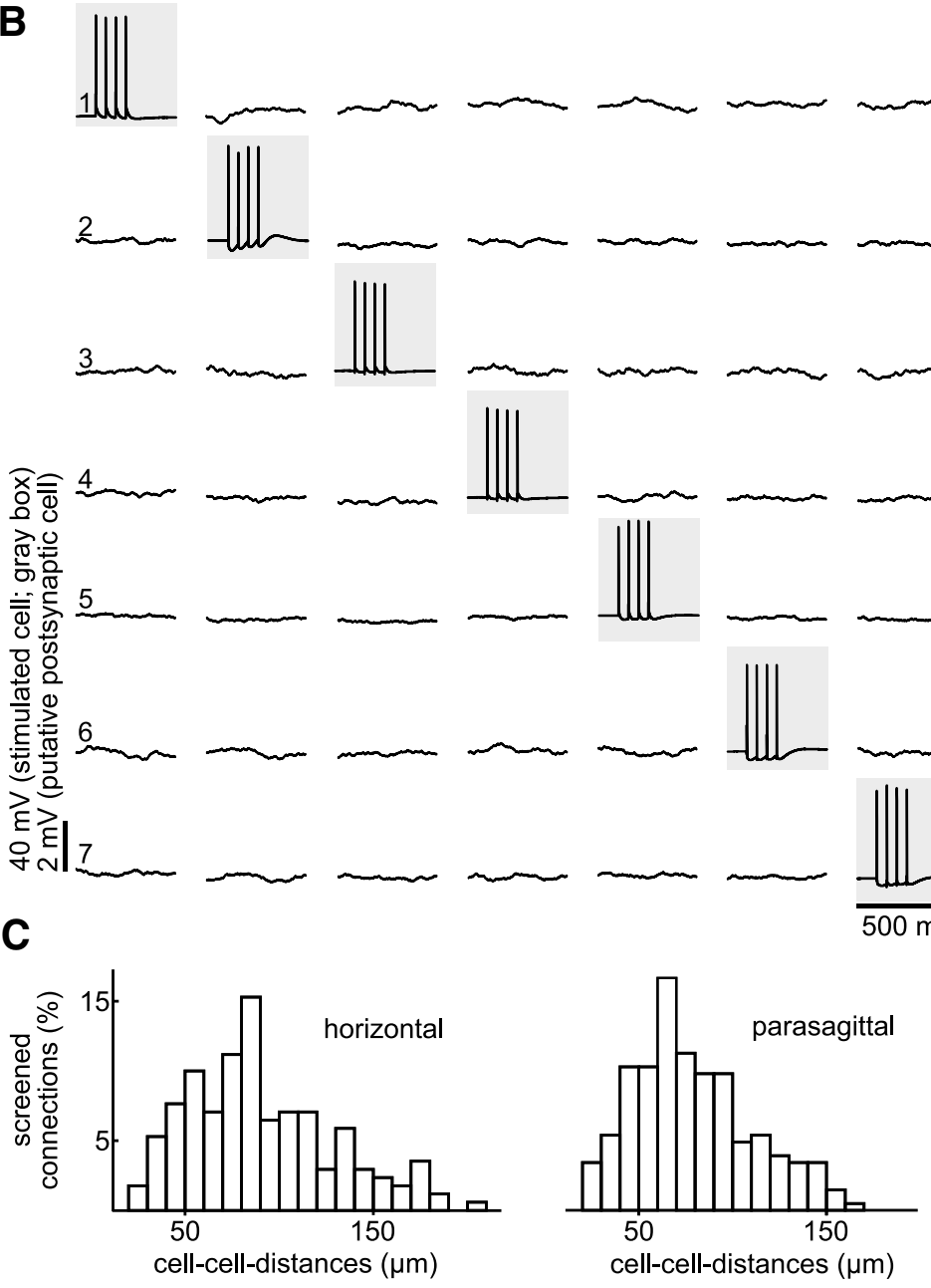

Statistical analysis. Statistical analyses were performed in Excel (Microsoft; RRID: SCR_016137) and MATLAB (The MathWorks; RRID:SCR_001622). Data are presented as the median and interquartile range, unless otherwise stated. Boxplots (central line, median; box, $25 \%-75 \%$; maximal whisker length, 2 times the interquartile range; data points beyond the whiskers displayed using "+") are used to illustrate sample distributions. Group data were compared using nonparametric Wilcoxon signed-rank test and Mann-Whitney $U$ test for paired and nonpaired comparisons, respectively. Multiple comparisons were corrected for by multiplying $p$ values by the number of comparisons (Bonferroni correction). Final $p$ values $<0.05$ were considered significant.

\section{Results}

Multipatch experiments reveal no synaptic interconnectivity between simultaneously recorded STN neurons To probe intranuclear mutual connectivity between STN neurons, we performed multiple whole-cell recordings from local clusters of up to 7 neurons simultaneously (Fig. 2A), allowing us to test up to 42 possible synaptic connections at once (Fig. $2 B)$. Using this approach, we examined a total of 830 connections between STN neurons in the juvenile cohort: 418 of those in slices cut in the parasagittal plane and 412 in the horizontal plane. Intersomatic distances between recorded cells ranged between 20 and $208 \mu \mathrm{m}$ (Fig. 2C). The connectivity analysis was extended to an adult cohort to control for agedependent effects, whereby an additional 44 synaptic connections were tested. In none of the tested connections did an AP in a putative presynaptic cell result in a temporally correlated postsynaptic potential in a simultaneously recorded cell, indicating a lack of functional connectivity between STN neurons.

To assess the morphological characteristics of the recorded cells, a subset of neurons were intracellularly filled, visualized, and reconstructed (38 STN cells in 7 clusters). Examined STN neurons had homogeneous morphological characteristics, featuring an ovoid soma and a bipolar dendritic tree with 4-6 primary dendrites (median: 5). Dendrites were aspiny, extended up to a distance of $322.2 \mu \mathrm{m}$ ( 272.5 to $393 \mu \mathrm{m})$ from the soma, and had a total length of $2191 \mu \mathrm{m}$ (median; $1768-$ $2865 \mu \mathrm{m}$ ). Somatodendritic domains were restricted to the STN, although occasionally individual dendrites were observed to extend outside the nucleus for shorter distances. For 27 neurons, an axon could be unequivocally identified. Most
Figure 2. Simultaneous recordings of up to 7 STN neurons show no mutual synaptic connectivity. $\boldsymbol{A}$, Reconstruction of simultaneously recorded STN neurons (different cluster than in Fig. 1). $\boldsymbol{B}$, Connectivity screening of the set of cells in $\boldsymbol{A}$. Neurons are stimulated consecutively to generate a burst of four APs (gray boxes). Recordings of the other, potentially postsynaptic, neurons are shown in the respective column as averages of 40 sweeps. Note the complete lack of AP-evoked unitary postsynaptic potentials. C, Distributions of distances between tested pairs of neurons in horizontal and parasagittal slices. 
cells showed a typical axonal morphology as previously described for STN projection neurons (Koshimizu et al., 2013): a thick initial segment followed by a T-shaped bifurcation with the secondary axon branches projecting rostrally and caudally, respectively ( 16 cells; Figs. $1 E$, 3 ). In some of these neurons $(n=8)$, the caudal axon branch could be followed as far as the $\mathrm{SNr}$ within the slice. In a few neurons (6 cells), local axonal collaterals were observed. The number of collaterals emerging from the primary and secondary axon was, however, low, and they showed little further branching (median number of collateral branches per cell was $3 ; 1-5$ ). Therefore, the extent of the axon local collaterals remained limited with a total length of $368 \mu \mathrm{m}$ (median; 315-659 $\mu \mathrm{m}, 6$ cells).

As the possibility of intranuclear synaptic contacts has been raised by previous anatomical studies (Hammond and Yelnik, 1983; Kita et al., 1983; Chang et al., 1984; Ammari et al., 2010; GoutyColomer et al., 2018), we examined the overlap of dendritic and axonal arborizations of the recorded neurons. Visual inspection revealed axo-dendritic proximities between neuron pairs of the recorded and visualized STN clusters (Fig. 3). To systematically analyze the existence of such potential contacts, axons and dendrites of the reconstructed cells were examined pairwise for locations of close proximity (see Materials and Methods). In 50 of 84 cell pairs examined, a total of 91 axo-dendritic proximities was found. Of these, 79 involved a primary axon and 12 small-caliber axon collaterals. Axodendritic proximities were found mostly proximally on the potential postsynaptic cell at a median distance of $87 \mu \mathrm{m}$ (41$146 \mu \mathrm{m}, 91$ potential contacts) measured along the dendrites from the soma. In addition, we also observed 12 axo-axonic proximities in 8 of the 84 examined cell pairs. Thus, the overlap of axonal and dendritic arbors and the existence of proximities between neurites of STN cells suggest that an anatomical potential of intranuclear connectivity exists; however, these spatial proximities do not translate into functional synaptic connections between the cells.

\section{Minimal stimulation experiments demonstrate sparse projections by incoming afferent fibers onto local clusters of STN neurons}

To study functional connectivity of afferents to STN neurons and in particular their divergence, we next applied extracellular stimulation while recording in voltage-clamp mode from clusters of STN neurons (Fig. 4). The stimulating electrode was placed in the
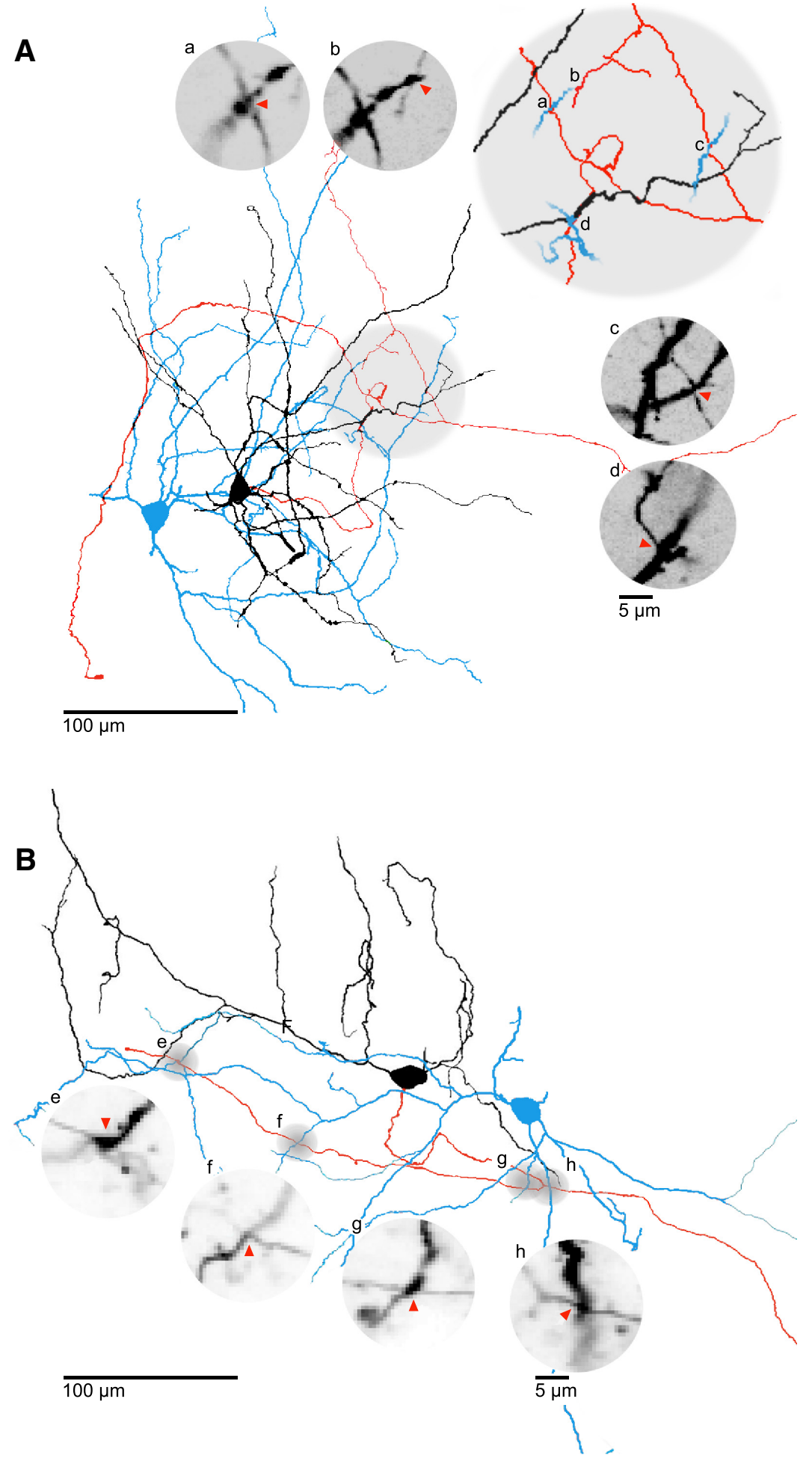

Figure 3. Local axo-dendritic proximities between neurons of the STN. $A, B, 2 D$ representations of reconstructed STN neurons with axo-dendritic proximities. Soma and dendrites of putative presynaptic cells (black) and their axons (red); blue represents putative postsynaptic cells. Gray circles represent site of proximity. Insets, Confocal z stack close-ups of the proximities. Letters a- $h$ show corresponding sites in $2 \mathrm{D}$ representations of reconstructed neurons. Red arrows indicate potential contact site. The cells in panel $\boldsymbol{A}$ are taken from the cluster displayed in Figure $1 E$. rostral end of the STN, where afferents enter the nucleus (Kita and Kita, 2012; Mallet et al., 2012). To activate single afferent axons, we applied a minimal stimulation protocol, and divergence was assessed by the number of simultaneously appearing 
A
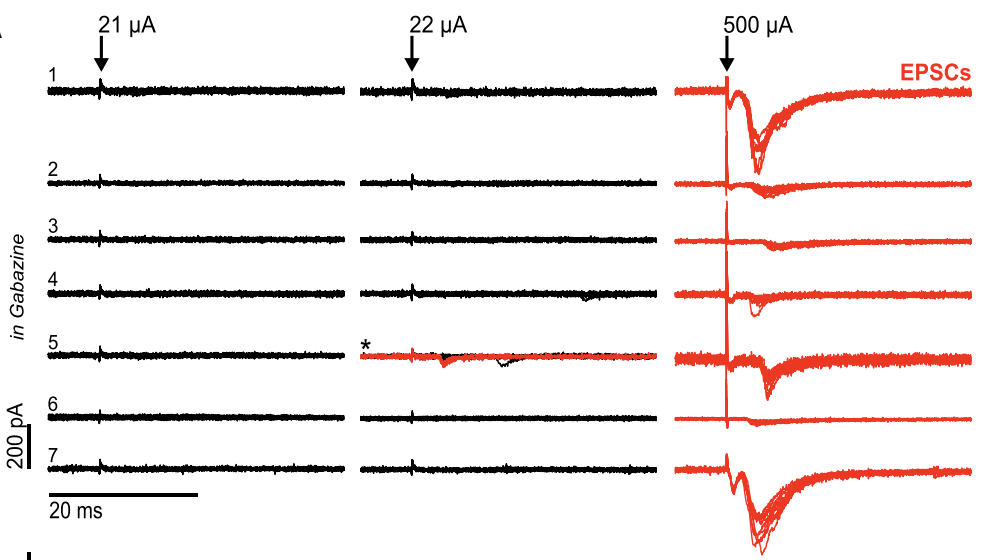

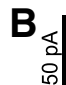
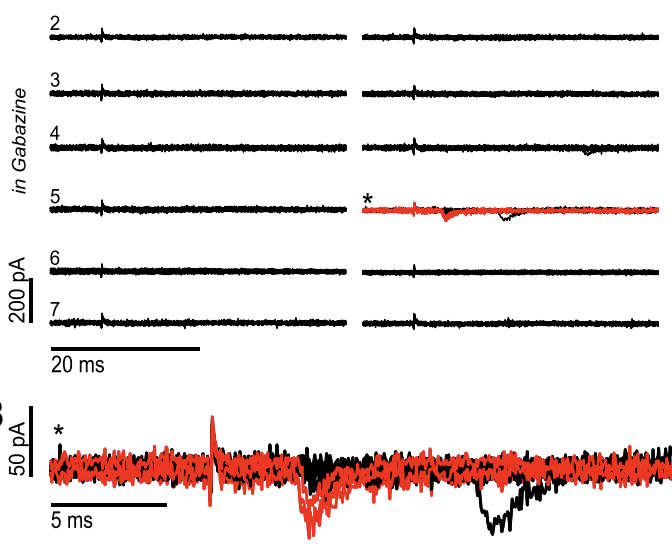

C
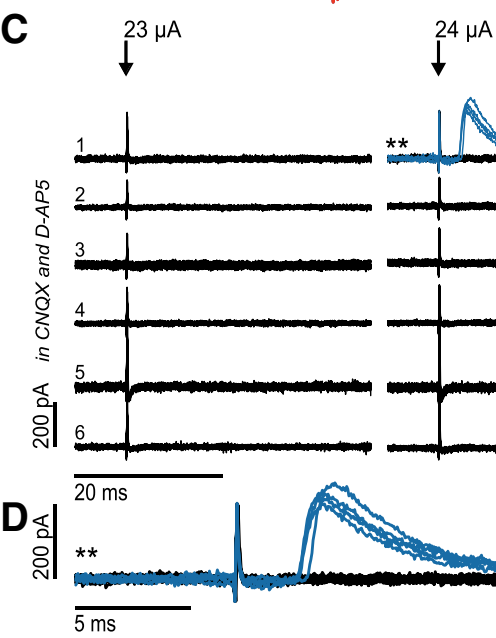

E
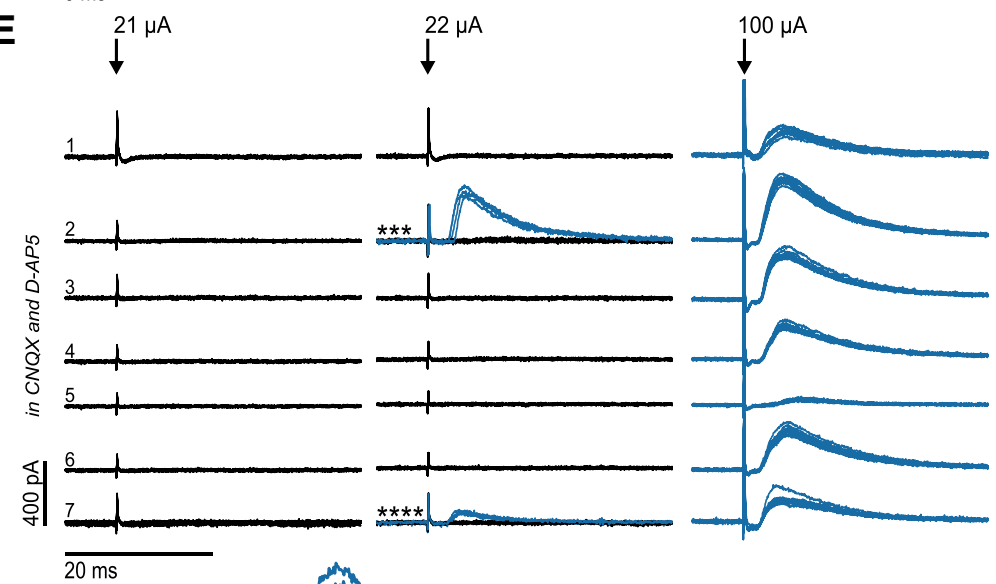

$\mathbf{F}$

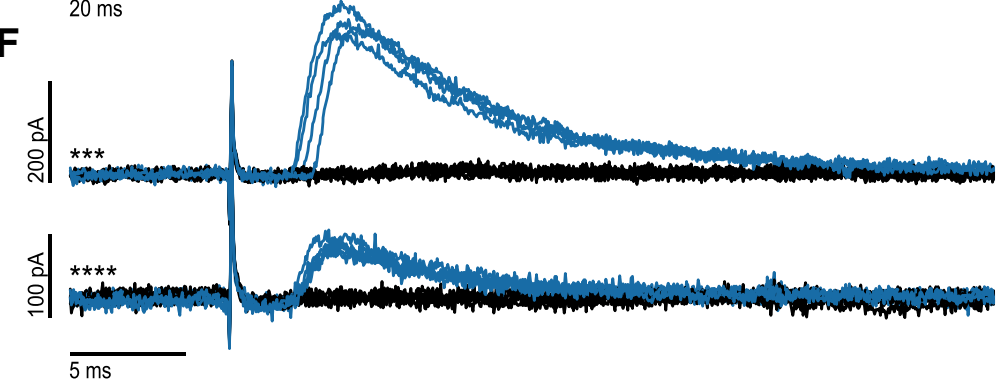

Figure 4. Minimal stimulation experiments reveal a low divergence of glutamatergic and GABAergic projections to local clusters of STN neurons. A, Minimal stimulation of afferents to a cluster of STN neurons in the presence of the $G A B A_{A}-R$ blocker synaptic inputs in cells of the recorded cluster (see Materials and Methods). Given our finding of absent intranuclear connectivity, responses to intranuclear stimulation should reflect the activation of projections to the nucleus.

In all cell clusters tested, the excitatory synaptic input was detected in only one of the recorded neurons at minimal stimulation intensity, and this was independent of the slice orientation (horizontal plane: 4 clusters; cluster sizes: 7, 7, 7, and 6 cells; parasagittal plane: 4 clusters; cluster sizes: $7,7,7$, and 6 cells; Fig. $4 A, B$ ), indicating a very low divergence of the input fibers. When stimulation intensity was subsequently increased, synaptic inputs were also recorded in other cells of the respective cluster, confirming that these cells also received afferent synaptic inputs; however, these can be assumed to be mediated by distinct afferent fibers.

This observation was replicated for the GABAergic input in the presence of CNQX and APV: in 7 of 10 clusters tested, only a single cell showed inhibitory synaptic responses at threshold stimulation intensity (horizontal plane: 4 clusters, cluster sizes: 7, 7, 6, and 5 cells; parasagittal plane: 3 clusters, cluster sizes: 5 , 5, and 5 cells; Fig. 4C,D). However, in 3 further clusters, postsynaptic currents were recorded in two neurons at the minimal stimulation intensity (horizontal plane: 2 clusters, cluster sizes: 7 and 4 cells; parasagittal plane: 1 cluster, cluster size: 6 cells; Fig. $4 E, F)$, reflecting that a sparse divergence of afferent fibers may exist.

GABAergic responses were elicited with short latency $(2.81 \mathrm{~ms}, 2.22-3.75 \mathrm{~ms}$, $n=13)$ in the presence of AMPA- and NMDA-receptor blockers, making polysynaptic activation unlikely. There was no difference between synaptic latencies of glutamatergic responses $(2.81 \mathrm{~ms}, 2.34-$ $4.0 \mathrm{~ms}, n=8$ ) and GABAergic responses. Thus, it is highly likely that both the IPSCs

$\leftarrow$

gabazine. *First neuron of the cluster that displayed evoked postsynaptic currents. Red represents traces in which evoked postsynaptic currents were detected. $\boldsymbol{B}$, Enlarged traces from neuron $5\left(^{*}\right)$ in $A$. C, Minimal stimulation of afferents to a cluster of STN neurons in the presence of CNQX and D-AP5 (glutamate-receptor blockers). ${ }^{*}$ First neuron of the cluster that displayed evoked postsynaptic currents. Blue represents traces in which evoked postsynaptic currents were detected. D, Enlarged traces from neuron $\left.1{ }^{(*)}\right)$ in C. E, Minimal stimulation of afferents to a cluster of STN neurons in the presence of CNQX and D-AP5 (glutamate-R blockers). Parallel appearance of two inhibitory synaptic inputs $\left(^{* * *},{ }^{* * * *}\right)$. Blue represents traces in which evoked postsynaptic currents were detected. $\boldsymbol{F}$, Enlarged traces from neuron $\left.2{ }^{(* * *}\right)$ and neuron $7\left(^{* * *}\right)$ in $\boldsymbol{E}$. 
and the EPSCs were monosynaptic. Furthermore, synaptic amplitudes of the glutamatergic responses were in a remarkably narrow range (27.5 mA, $24.75-31.5 \mathrm{~mA}, n=8$ ), consistent with their single contact-mediated, monosynaptic nature. GABAergic inputs, in contrast, had large amplitudes with higher variability ( 47 $\mathrm{mA}, 22.38-110.63 \mathrm{~mA}, n=13$ ). This finding is consistent with previously reported sparsely distributed terminal clusters of GABAergic afferent input to the STN that form multiple synaptic contacts onto individual STN neurons (Baufreton et al., 2009).

In summary, this suggests that both glutamatergic and GABAergic incoming fibers to the nucleus provide a sparse and selective, rather than a broad and divergent, input onto local clusters of STN neurons.

\section{Costimulation of inhibitory and excitatory afferents delays and disperses AP generation in STN neurons}

To investigate the joint effect of convergent glutamatergic and GABAergic afferents, large-intensity extracellular stimuli were applied (see Materials and Methods). The simultaneous activation of the sparse projections produced compound postsynaptic currents in individual STN neurons (Fig. 5). Within the same cell cluster, however, neurons showed diverse synaptic responses predominantly glutamatergic, GABAergic, or mixed (Fig. $5 B$ ).

To study the timing of AP generation in STN neurons in response to these variable synaptic currents, we switched to the current-clamp mode (Fig. $5 C$ ). Under this condition, the same stimuli elicited APs in a subset of cells (32 of 142 neurons tested, 23\%). In most cells, the APs had long latencies and showed high temporal dispersion (Fig. 5C). On the group level, the median latency was $20.2 \mathrm{~ms}(6.81-119.8 \mathrm{~ms}, n=32)$, with a temporal dispersion of $7.24 \mathrm{~ms}$ (SDs across cells, $0.77-31 \mathrm{~ms}, n=28$ ).

To next examine APs evoked by the excitatory input in isolation, we bath-applied gabazine to block $\mathrm{GABA}_{\mathrm{A}}$ receptormediated synaptic components (Fig. 5D). This manipulation resulted in a recruitment of $32 \%$ of STN neurons ( 23 of 73 neurons tested). Compared with control, APs were elicited with markedly shorter latency (median latencies across cells: $7.3 \mathrm{~ms}$, 4.53-8.6 ms, $n=22 ; p=0.002$ ) and lower temporal variability (SDs across cells: $0.46 \mathrm{~ms}, 0.12-1.11 \mathrm{~ms}, n=18 ; p=0.002$ ).

In summary, excitatory afferents alone drive STN neurons with short latency and low temporal variability. In contrast, costimulation of inhibitory afferents delays and disperses AP generation in STN neurons. Thus, the interaction of simultaneously recruited glutamatergic and GABAergic inputs can desynchronize neurons of the STN.

\section{Repetitive DBS-like stimulation produces differential short-term depression of glutamatergic and GABAergic inputs to the STN}

In view of the tight interplay of glutamatergic and GABAergic inputs in the recruitment of STN neurons, we next aimed to study the dynamics of these two afferent systems during repetitive stimulation. We applied $1 \mathrm{~s}$ trains of extracellular stimuli at low (10 and $20 \mathrm{~Hz})$ and high, DBS-like frequencies $(130 \mathrm{~Hz})$ while recording from the cell clusters (Fig. 6). As described above, the compound synaptic responses were variable across the cells of the recorded clusters. Nevertheless, both compound glutamatergic and GABAergic synaptic responses showed short-term depression during the stimulus train.

For low-stimulation frequencies, synaptic depression was comparable for compound excitatory and compound inhibitory responses. At $10 \mathrm{~Hz}$, the synaptic depression ratio (fifth/first synaptic amplitude) for EPSCs was $0.44(0.4-0.55, n=12)$ and for
IPSCs $0.58(0.55-0.62, n=9 ; p=0.27$ for EPSCs vs IPSCs). At 20 $\mathrm{Hz}$, the depression ratio for EPSCs was $0.32(0.3-0.54, n=12)$ and for IPSCs $0.58(0.5-0.62, n=9 ; p=0.46$ for EPSCs vs IPSCs; Fig. 6A). The synaptic depression ratios were not significantly different between $10 \mathrm{~Hz}$ and $20 \mathrm{~Hz}$ stimulation neither for compound EPSCs $(p=0.61)$ nor for IPSCs $(p=1)$. However, DBSlike high-frequency stimulation at $130 \mathrm{~Hz}$ caused a dramatic decrease of the EPSC amplitude over the train resulting in a depression ratio of $0.07(0.03-0.13, n=12$; Fig. $6 B, C)$. Comparing the degree of depression of EPSCs, the difference was highly significant between $130 \mathrm{~Hz}$ and $10 \mathrm{~Hz}(p=0.004)$ or $20 \mathrm{~Hz}(p=$ 0.004 ), respectively (Fig. $6 D$ ). In contrast, the IPSCs showed only moderate depression even at high frequencies (ratio of 0.46, 0.4 $0.48, n=9 ; p=0.001$ for EPSCs vs IPSCs) and no statistical differences when comparing stimulation frequencies of $130 \mathrm{~Hz}$ versus $10 \mathrm{~Hz}(p=0.07)$ or $20 \mathrm{~Hz}$ ( $p=0.18$; Fig. $6 D)$.

Despite strong synaptic depression during the stimulation train, both EPSCs and IPSCs rapidly recovered after a $4 \mathrm{~s}$ break (Fig. 6E). Synaptic amplitudes in response to the first stimulus in the trains showed no significant difference between the first and the 10th repetition either for EPSCs (first: $-78 \mathrm{pA},-118$ to -62 pA; 10th: $-85 \mathrm{pA},-125$ to $-52 \mathrm{pA}, n=12 ; p=0.57$ ) or for IPSCs (first: $109 \mathrm{pA}, 67$ to $226 \mathrm{pA}$; 10th: $130 \mathrm{pA}, 75$ to $237 \mathrm{pA}$, $n=9 ; p=0.91$; Fig. $6 F$ ).

In summary, the degree of synaptic depression does not differ between compound glutamatergic and GABAergic synaptic inputs for low-stimulation frequencies. However, at high, DBS-like frequencies, the compound glutamatergic drive rapidly and almost completely depresses after only a few stimuli in the train. In contrast, the GABAergic input remains relatively robust at a moderate level of depression. Thus, the differential dynamics of STN inputs will cause a major shift in the balance of excitation and inhibition toward inhibition.

\section{Discussion}

In our study, focusing on the synaptic connectivity of the STN, we found no evidence for intranuclear mutual connections between STN neurons in acute slices of juvenile and adult rats. We observed sparse divergence of individual afferent fibers of both glutamatergic and GABAergic input onto neurons of the recorded clusters when tested by a minimal stimulation protocol. Recruitment of pharmacologically isolated glutamatergic afferents at higher stimulus intensities evoked short-latency, highly synchronous APs, whereas costimulation of glutamatergic and GABAergic afferents resulted in delayed and dispersed AP generation in STN neurons. Finally, repetitive extracellular stimulation at high, DBS-like frequencies, but not at low frequencies, produced differential short-term plasticity of glutamatergic and GABAergic inputs, due to a dramatic reduction of excitatory but not inhibitory responses. Thus, DBS-like stimulation patterns can dynamically shift the balance of synaptic excitation and inhibition in the STN toward inhibition.

\section{Sparse afferent and absent intrinsic connectivity of the STN}

The rat STN is considered to comprise a homogeneous population of glutamatergic neurons. Nevertheless, features of GABAergic transmission in a subset of STN neurons have previously been reported despite predominant evidence for their glutamatergic phenotype (Jin et al., 2011). Lévesque and Parent (2005) further suggested that the human STN contains GABAergic interneurons. The lack of vGAT-YFP expression and the homogeneity of morphological properties in our sample do not support the existence of GABAergic neurons in the 


\section{A}

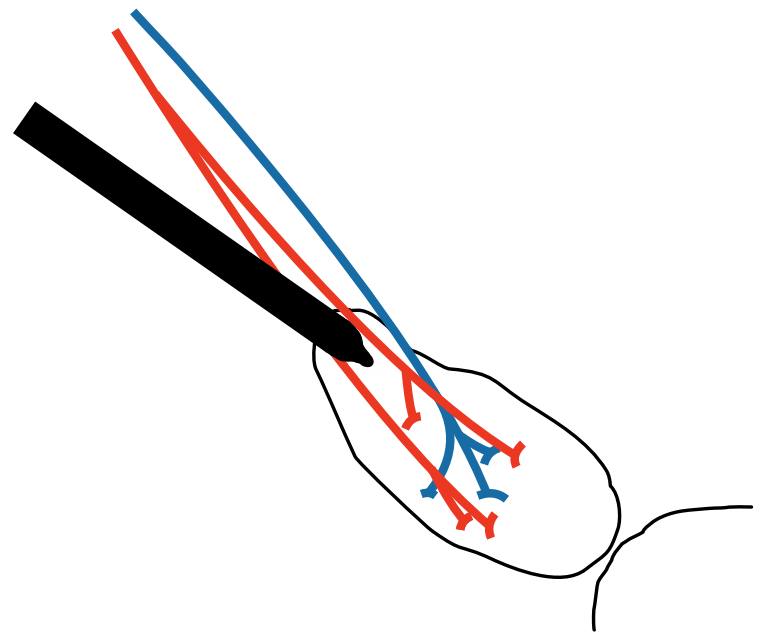

B
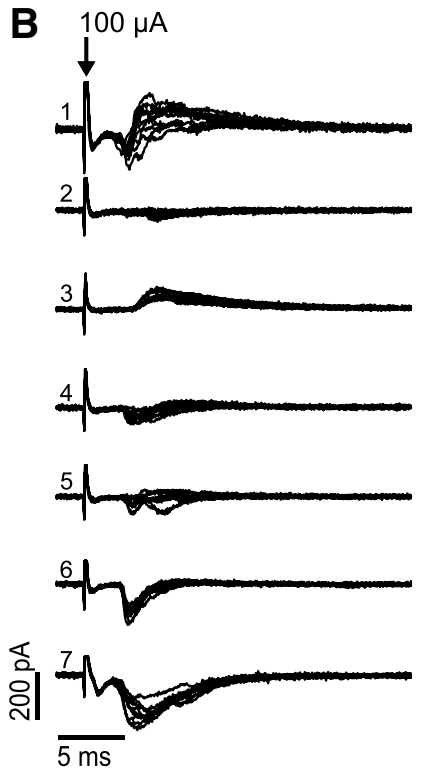

C
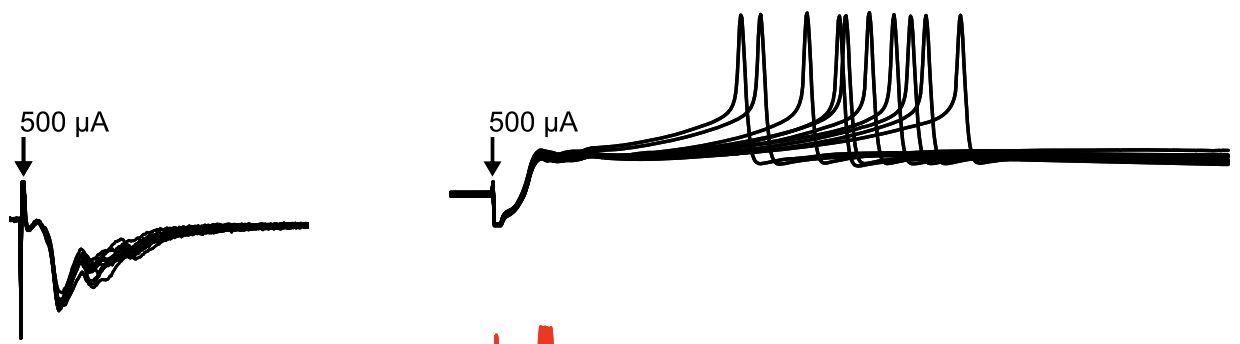

D in Gabazine
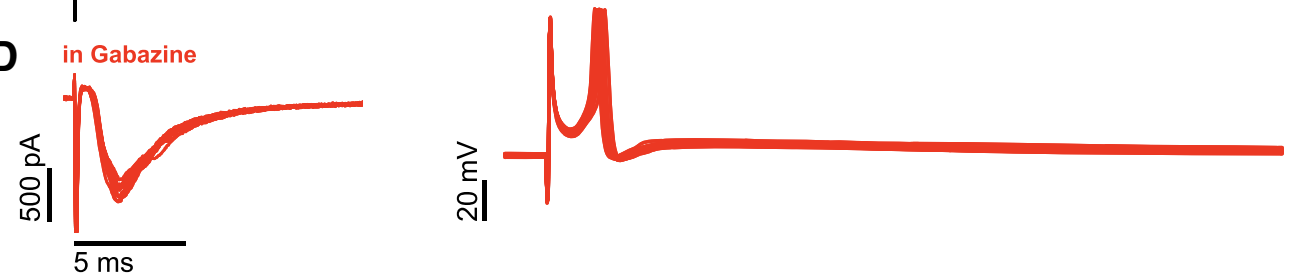

$\mathbf{E}$
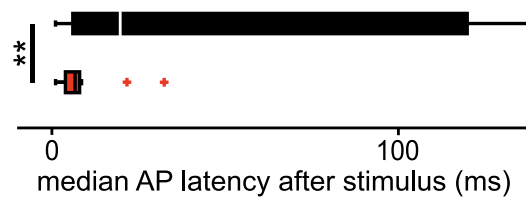

$\mathbf{F}$

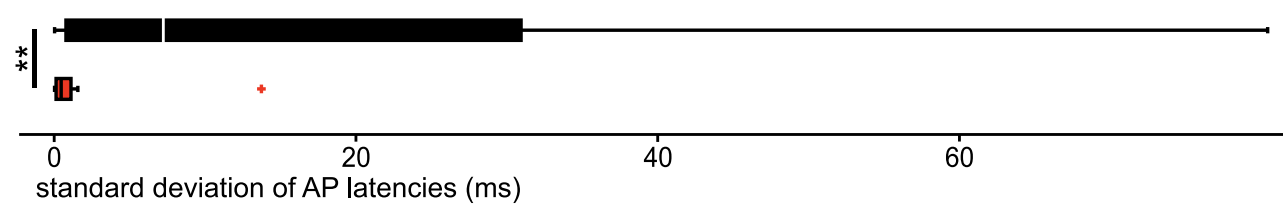

Figure 5. GABAergic costimulation delays and desynchronizes AP generation in STN neurons. $A$, Schematic drawing of experimental paradigm. Red represents glutamatergic afferents to the STN. Blue represents GABAergic afferents to the STN. B, Example traces of compound glutamatergic, GABAergic, and mixed compound synaptic currents in a cluster of STN neurons. C, Voltage-clamp and current-clamp recordings of an individual STN neuron. $\boldsymbol{D}$, Voltage-clamp and current-clamp recordings of the same STN neuron as in $\boldsymbol{C}$ in the presence of gabazine. $\boldsymbol{E}$, Boxplots displaying the distribution of the medians of AP latency following a single stimulus as exemplified in $\mathbf{C}$ and $\boldsymbol{D}$. Top, $n=32$ neurons without synaptic blockers (black). Bottom, $n=22$ in the presence of gabazine (red). ${ }^{*}$ Highly significant difference $(p<0.01)$. $\boldsymbol{F}$, Boxplots displaying the distribution of SDs of AP latency after a single stimulus as exemplified in $\boldsymbol{C}$ and $\boldsymbol{D}$. Top, $n=28 \mathrm{neurons}$ without synaptic blockers (black). Bottom, $n=18$ in the presence of gabazine (red).

rat STN. This question, however, would need further investigation and the possibility of interspecies differences has to be acknowledged.

Intranuclear mutual connectivity between glutamatergic STN neurons has been proposed to be of crucial importance for com- putational function and to contribute to neural synchrony in this nucleus (Gillies and Willshaw, 2004; Shen and Johnson, 2006; Ammari et al., 2010). Convergently, anatomical studies demonstrated local axon collaterals of STN neurons (Hammond and Yelnik, 1983; Kita et al., 1983; Chang et al., 1984; Ammari et al., 


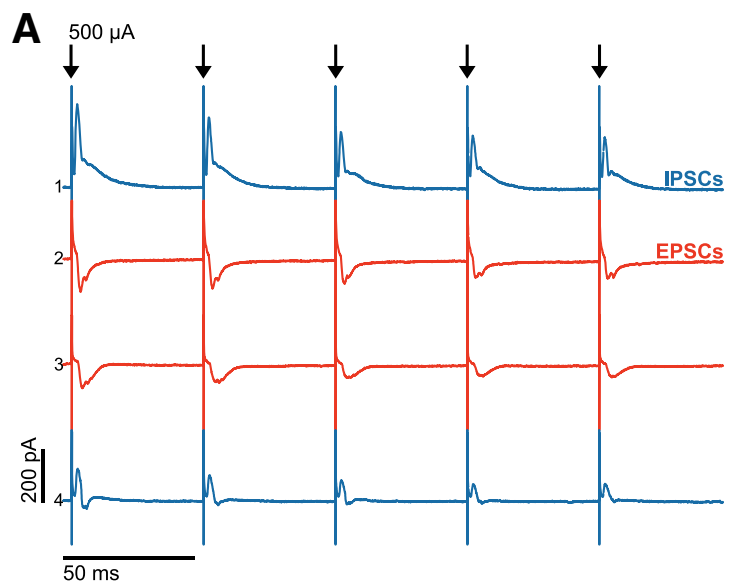

C

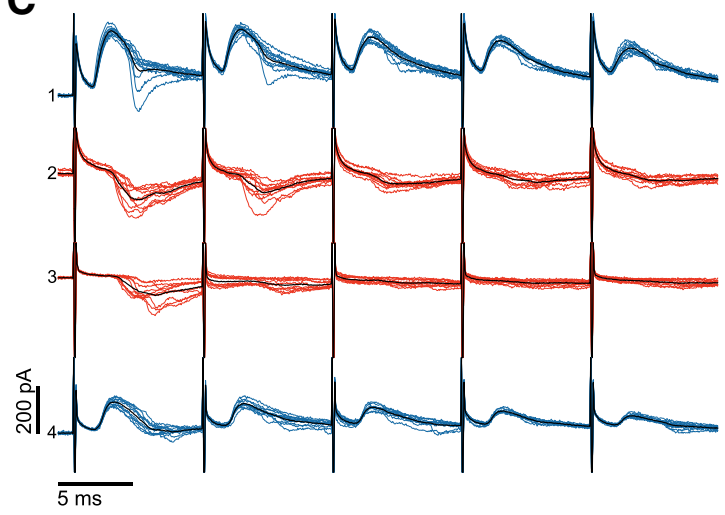

E ${ }^{1 \text { sstim of }}{ }^{1 s}$ stim of

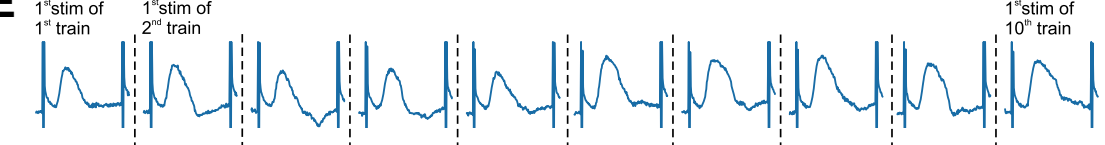

各
B 500 H

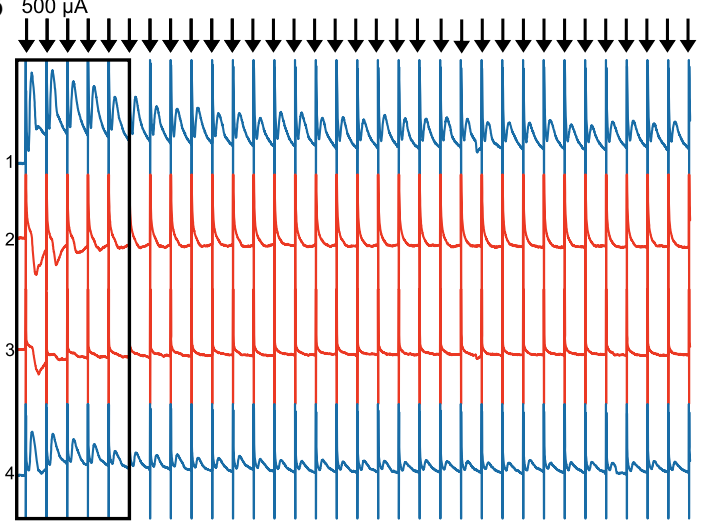

D

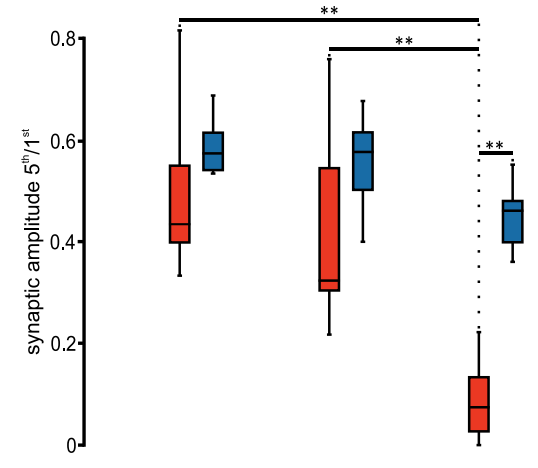

$20 \mathrm{~Hz} \quad 130 \mathrm{~Hz}$

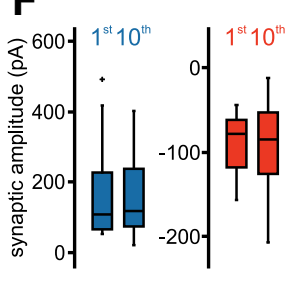

Figure 6. Sustained inhibition during DBS-like stimulation contrasts rapid depression of excitatory inputs. $A$, Averaged voltage-clamp traces at $20 \mathrm{~Hz}$ stimulation frequency in four simultaneously recorded STN neurons. $\boldsymbol{B}$, Averaged voltage-clamp traces from the same STN neurons as in $\boldsymbol{A}$ at $130 \mathrm{~Hz}$ stimulation frequency. $\boldsymbol{C}$, Same traces as in $\boldsymbol{B}$ (black frame), albeit as single sweeps at higher temporal resolution; the respective average traces are superimposed in black. $D$, Group data ( $n=12$ compound, predominantly glutamatergic EPSCs: red boxes; and $n=9$ compound, predominantly GABAergic IPSCS: blue boxes) show distribution of synaptic depression ratios (fifth/first) for the respective stimulation frequencies. ${ }^{*}$ Highly significant difference $(p<0.01)$. $\boldsymbol{E}$, Representative traces show the synaptic responses evoked by the first pulses of the 10 consecutive $130 \mathrm{~Hz}$ stimulus trains. Blue represents compound, predominantly GABAergic IPSCs. Red represents predominantly glutamatergic EPSCS. $\boldsymbol{F}$, Summary box charts show the peak amplitudes of the first synaptic responses in the first and 10th stimulation trains. Blue represents compound, predominantly GABAergic IPSCS $(n=9)$. Red represents compound, predominantly glutamatergic $\operatorname{ESSCS}(n=12)$.

2010; Gouty-Colomer et al., 2018), interpreted as indication of local synaptic connections. Consistently, we found axon collaterals, albeit with a limited extent, for a subset of STN neurons. We also observed proximities between axons and dendrites of STN neurons. Results of our extensive multiple whole-cell recordings, however, revealed no functional connectivity between any of the closely spaced neuron pairs examined. Thus, the putative anatomical contacts do not translate into discernible functional connectivity, making mutual synaptic connectivity between glutamatergic STN neurons unlikely. This is in good agreement with previous acute slice work that investigated synchrony in the STN (Wilson et al., 2004). Our results contrast those obtained in organotypic rat midbrain slice culture pointing to intranuclear excitatory connectivity (Chu et al., 2012), which, however, might be explained by extensive axonal sprouting in such preparations (Debanne et al., 1998). Local axon collaterals and axo-dendritic proximities may serve other functions, such as regulation of remote inputs, as suggested for striatal microcircuits (Du et al., 2017).

Combination of multiple recordings with minimal stimulation further allowed us to assess the divergence of afferent inputs to the STN. Our results reveal that both excitatory and inhibitory afferents show low divergence onto the closely spaced neurons of STN cell clusters. This finding is in good agreement with a previous study demonstrating a sparse and selective connectivity from the GPe onto STN neurons (Baufreton et al., 2009). Our results extend these findings by showing that glutamatergic projections exhibit a similar or even more selective projection pattern, in good agreement with previous anatomical data (Kita and Kita, 2012).

Both mutual intrinsic and divergent afferent connectivity may serve as anatomical substrates for the emergence of synchrony in a local neuronal network. Although we cannot fully rule out the 
existence of recurrent excitatory connections in the STN, these must be of very low occurrence with minimal somatic effect. Furthermore, the afferent inputs, in particular the excitatory projections, show minimal divergence. Together, these features identify STN neurons as independent processing units, which integrate convergent sparse afferent inputs in a largely parallel manner. Synchrony in such a system will be inherited from upstream structures. Reciprocal interactions with the GPe may further endow the STN with pattern generation capabilities (Bevan et al., 2002). Because remote afferents take center stage for the synchronization in the STN, their interactions and dynamic properties are critical for synaptic control of the nucleus.

\section{GABAergic coactivation desynchronizes STN neurons}

Activation of the afferent input at higher stimulation intensities produced diverse patterns of compound synaptic currents across the neurons of the recorded clusters, reflecting the convergence of a varying composition of stimulated glutamatergic and GABAergic fibers onto the cells. While the convergent excitatory input in isolation was able to recruit STN neurons with short latency and high temporal precision, the costimulation of GABAergic fibers delayed and desynchronized APs under control conditions, as suggested by Bevan et al. (2007).

However, simultaneous convergent glutamatergic and GABAergic input is unlikely to occur under in vivo conditions. Still, the extracellular costimulation paradigm of excitatory and inhibitory afferents in our experiments might bear direct relevance for DBS in the clinical setting, which similarly activates a mixed population of afferent fibers (Reich et al., 2015). In PD, pallidal GABAergic transmission to the STN is increased (Fan et al., 2012; Chu et al., 2015) and phase-shifted (Mallet et al., 2008a,b) relative to synchronized motor cortex excitation (Goldberg et al., 2002). Importantly, out-of-phase inhibition, when preceding EPSPs, can support AP generation by increasing the availability of $\mathrm{Na}^{+}$ channels (Baufreton et al., 2005). In an oscillatory context, when excitation and inhibition alternate in the cycle, inhibition can enhance the efficiency, precision, and ultimately the synchrony of spiking (Baufreton et al., 2005). Our results on the desynchronizing effect of GABAergic costimulation suggest that DBS inverts this synchronizing role of the GABAergic input. In forcing simultaneous activation of inhibitory and excitatory inputs, it is directly shunting and hyperpolarizing excitatory input, effectively closing the time window in which a synchronized afferent drive can recruit the STN.

\section{Sustained inhibition contrasts rapid depression of excitatory inputs during high-frequency stimulation}

The particular susceptibility of the glutamatergic drive to synaptic depression upon repetitive stimulation at high frequencies has further relevance for DBS. Previous studies have proposed shortterm depression as a DBS mechanism of action (Zheng et al., 2011; Rosenbaum et al., 2014; Milosevic et al., 2018). Our results corroborate this finding: in contrast to stimulation at low frequencies, when glutamatergic and GABAergic inputs showed comparable degrees of depression, high frequencies produced a rapid and dramatic reduction in EPSCs, whereas IPSCs remain relatively robust, resulting in a shift toward inhibition. Noteworthy, the activation of inhibitory input during DBS has recently received attention (Chiken and Nambu, 2014), but has not been directly compared to DBS effects on excitatory inputs. The shift in the balance largely decouples the STN from its glutamatergic cortical drive, whereas the maintained inhibitory input promotes desynchronization. Thus, differential short-term depression of synaptic transmission represents a mechanism to attenuate the unpredictable variability of recruited afferent fibers.

\section{From synaptic dynamics to therapeutic interventions}

While the STN receives glutamatergic and GABAergic inputs, previous studies argued that the selective stimulation of the hyperdirect pathway alone could account for therapeutic effects of DBS (Gradinaru et al., 2009; Sanders and Jaeger, 2016). Both the therapeutic DBS effects observed in the aforementioned studies and the effects on glutamatergic afferent control presented in our study critically depend on stimulation frequency. Limiting the DBS mechanism of action to effects on the hyperdirect pathway, however, fails to explain why electrode placement in the GPi, a frequently used alternative target in PD which is not monosynaptically connected to the cortex, is comparable in its clinical effect (Follett et al., 2010; Williams et al., 2014). Indeed, recent studies suggest that GPi-DBS, similar to STN-DBS, is reducing cortical beta-gamma phase-amplitude coupling (Malekmohammadi et al., 2018), a previously reported biomarker of PD (de Hemptinne et al., 2013). Thus, it seems unlikely that the DBS mechanism of action is exclusively depending on selective stimulation of the hyperdirect pathway. Instead, electrical stimulation plausibly targets both GABAergic and glutamatergic fibers simultaneously. As both GPi and STN are similar in integrating GABAergic and glutamatergic inputs, differential short-term plasticity could provide a general, target-independent mechanism that prevents the downstream propagation of pathological activity.

Further, our results may shed light on the synaptic mechanisms underlying feedback-controlled intermittent forms of DBS (Little et al., 2013; Herz et al., 2018). These adaptive stimulation paradigms have been proposed to trim excessive synchronization at beta frequencies with high temporal precision (RamirezZamora et al., 2017; Tinkhauser et al., 2017b). Our data suggest that the rapid depression of glutamatergic inputs and the promotion of desynchronizing afferent control complies with the necessity for fast, temporally precise disruption of local synchrony. Because synaptic recovery is complete within seconds, intermittent DBS allows for physiological synchronization dynamics in interstimulation intervals. In conclusion, the dynamic properties of afferent synapses revealed in this study fit well to intermittent stimulation paradigms, allowing for fast yet temporally restricted functional decoupling of the STN from synchronizing inputs.

\section{References}

Ammari R, Lopez C, Bioulac B, Garcia L, Hammond C (2010) Subthalamic nucleus evokes similar long lasting glutamatergic excitations in pallidal, entopeduncular and nigral neurons in the basal ganglia slice. Neuroscience 166:808-818.

Baufreton J, Atherton JF, Surmeier DJ, Bevan MD (2005) Enhancement of excitatory synaptic integration by GABAergic inhibition in the subthalamic nucleus. J Neurosci 25:8505-8517.

Baufreton J, Kirkham E, Atherton JF, Menard A, Magill PJ, Bolam JP, Bevan MD (2009) Sparse but selective and potent synaptic transmission from the globus pallidus to the subthalamic nucleus. J Neurophysiol 102:532545.

Benabid AL, Pollak P, Gross C, Hoffmann D, Benazzouz A, Gao DM, Laurent A, Gentil M, Perret J (1994) Acute and long-term effects of subthalamic nucleus stimulation in Parkinson's disease. Stereotact Funct Neurosurg 62:76-84.

Bevan MD, Hallworth NE, Baufreton J (2007) GABAergic control of the subthalamic nucleus. Prog Brain Res 160:173-188.

Bevan MD, Magill PJ, Terman D, Bolam JP, Wilson CJ (2002) Move to the rhythm: oscillations in the subthalamic nucleus-external globus pallidus network. Trends Neurosci 25:525-531. 
Böhm C, Peng Y, Maier N, Winterer J, Poulet JF, Geiger JR, Schmitz D (2015) Functional diversity of subicular principal cells during hippocampal ripples. J Neurosci 35:13608-13618.

Bolam JP, Hanley JJ, Booth PA, Bevan MD (2000) Synaptic organisation of the basal ganglia. J Anat 196:527-542.

Chang HT, Kita H, Kitai ST (1984) The ultrastructural morphology of the subthalamic-nigral axon terminals intracellularly labeled with horseradish peroxidase. Brain Res 299:182-185.

Chen CC, Brücke C, Kempf F, Kupsch A, Lu CS, Lee ST, Tisch S, Limousin P, Hariz M, Brown P (2006) Deep brain stimulation of the subthalamic nucleus: a two-edged sword. Curr Biol 16:R952-R953.

Chiken S, Nambu A (2014) Disrupting neuronal transmission: mechanism of DBS? Front Syst Neurosci 8:33.

Chu HY, Atherton JF, Wokosin D, Surmeier DJ, Bevan MD (2015) Heterosynaptic regulation of external globus pallidus inputs to the subthalamic nucleus by the motor cortex. Neuron 85:364-376.

Chu JU, Jeong MJ, Song KI, Lee HC, Kim J, Kim YJ, Choi K, Suh JK, Youn I (2012) Spontaneous synchronized burst firing of subthalamic nucleus neurons in rat brain slices measured on multi-electrode arrays. Neurosci Res 72:324-340.

Debanne D, Gähwiler BH, Thompson SM (1998) Long-term synaptic plasticity between pairs of individual CA3 pyramidal cells in rat hippocampal slice cultures. J Physiol 507:237-247.

de Hemptinne C, Ryapolova-Webb ES, Air EL, Garcia PA, Miller KJ, Ojemann JG, Ostrem JL, Galifianakis NB, Starr PA (2013) Exaggerated phase-amplitude coupling in the primary motor cortex in Parkinson disease. Proc Natl Acad Sci U S A 110:4780-4785.

Du K, Wu YW, Lindroos R, Liu Y, Rózsa B, Katona G, Ding JB, Kotaleski JH (2017) Cell-type-specific inhibition of the dendritic plateau potential in striatal spiny projection neurons. Proc Natl Acad Sci U S A 114:E7612E7621.

Fan KY, Baufreton J, Surmeier DJ, Chan CS, Bevan MD (2012) Proliferation of external globus pallidus-subthalamic nucleus synapses following degeneration of midbrain dopamine neurons. J Neurosci 32:13718-13728.

Feingold J, Gibson DJ, DePasquale B, Graybiel AM (2015) Bursts of beta oscillation differentiate postperformance activity in the striatum and motor cortex of monkeys performing movement tasks. Proc Natl Acad Sci U S A 112:13687-13692.

Follett KA, Weaver FM, Stern M, Hur K, Harris CL, Luo P, Marks WJ Jr, Rothlind J, Sagher O, Moy C, Pahwa R, Burchiel K, Hogarth P, Lai EC, Duda JE, Holloway K, Samii A, Horn S, Bronstein JM, Stoner G, et al. (2010) Pallidal versus subthalamic deep-brain stimulation for Parkinson's disease. N Engl J Med 362:2077-2091.

Gillies A, Willshaw D (2004) Models of the subthalamic nucleus: the importance of intranuclear connectivity. Med Eng Phys 26:723-732.

Goldberg JA, Boraud T, Maraton S, Haber SN, Vaadia E, Bergman H (2002) Enhanced synchrony among primary motor cortex neurons in the 1-methyl-4-phenyl-1,2,3,6-tetrahydropyridine primate model of Parkinson's disease. J Neurosci 22:4639-4653.

Gouty-Colomer LA, Michel FJ, Baude A, Lopez-Pauchet C, Dufour A, Cossart R, Hammond C (2018) Mouse subthalamic nucleus neurons with local axon collaterals. J Comp Neurol 526:275-284.

Gradinaru V, Mogri M, Thompson KR, Henderson JM, Deisseroth K (2009) Optical deconstruction of parkinsonian neural circuitry. Science 324: 354-359.

Hammond C, Yelnik J (1983) Intracellular labelling of rat subthalamic neurones with horseradish peroxidase: computer analysis of dendrites and characterization of axon arborization. Neuroscience 8:781-790.

Herz DM, Little S, Pedrosa DJ, Tinkhauser G, Cheeran B, Foltynie T, Bogacz $\mathrm{R}$, Brown P (2018) Mechanisms underlying decision-making as revealed by deep-brain stimulation in patients with Parkinson's disease. Curr Biol 28:1169-1178.e6.

Jin XT, Galvan A, Wichmann T, Smith Y (2011) Localization and function of GABA transporters GAT-1 and GAT-3 in the basal ganglia. Front Syst Neurosci 5:63.

Kita H, Chang HT, Kitai ST (1983) The morphology of intracellularly labeled rat subthalamic neurons: a light microscopic analysis. J Comp Neurol 215:245-257.

Kita T, Kita H (2012) The subthalamic nucleus is one of multiple innervation sites for long-range corticofugal axons: a single-axon tracing study in the rat. J Neurosci 32:5990-5999.

Koshimizu Y, Fujiyama F, Nakamura KC, Furuta T, Kaneko T (2013) Quan- titative analysis of axon bouton distribution of subthalamic nucleus neurons in the rat by single neuron visualization with a viral vector. J Comp Neurol 521:2125-2146.

Kühn AA, Kempf F, Brücke C, Gaynor Doyle L, Martinez-Torres I, Pogosyan A, Trottenberg T, Kupsch A, Schneider GH, Hariz MI, Vandenberghe W, Nuttin B, Brown P (2008) High-frequency stimulation of the subthalamic nucleus suppresses oscillatory beta activity in patients with Parkinson's disease in parallel with improvement in motor performance. J Neurosci 28:6165-6173.

Lévesque JC, Parent A (2005) GABAergic interneurons in human subthalamic nucleus. Mov Disord 20:574-584.

Li Q, Ke Y, Chan DC, Qian ZM, Yung KK, Ko H, Arbuthnott GW, Yung WH (2012) Therapeutic deep brain stimulation in parkinsonian rats directly influences motor cortex. Neuron 76:1030-1041.

Little S, Pogosyan A, Neal S, Zavala B, Zrinzo L, Hariz M, Foltynie T, Limousin P, Ashkan K, FitzGerald J, Green AL, Aziz TZ, Brown P (2013) Adaptive deep brain stimulation in advanced Parkinson disease. Ann Neurol 74:449-457.

Longair MH, Baker DA, Armstrong JD (2011) Simple neurite tracer: open source software for reconstruction, visualization and analysis of neuronal processes. Bioinformatics 27:2453-2454.

Malekmohammadi M, AuYong N, Ricks-Oddie J, Bordelon Y, Pouratian N (2018) Pallidal deep brain stimulation modulates excessive cortical high beta phase amplitude coupling in Parkinson disease. Brain Stimul 11:607617.

Mallet N, Pogosyan A, Márton LF, Bolam JP, Brown P, Magill PJ (2008a) Parkinsonian beta oscillations in the external globus pallidus and their relationship with subthalamic nucleus activity. J Neurosci 28:1424514258.

Mallet N, Pogosyan A, Sharott A, Csicsvari J, Bolam JP, Brown P, Magill PJ (2008b) Disrupted dopamine transmission and the emergence of exaggerated beta oscillations in subthalamic nucleus and cerebral cortex. J Neurosci 28:4795-4806.

Mallet N, Micklem BR, Henny P, Brown MT, Williams C, Bolam JP, Nakamura KC, Magill PJ (2012) Dichotomous organization of the external globus pallidus. Neuron 74:1075-1086.

Milosevic L, Kalia SK, Hodaie M, Lozano AM, Fasano A, Popovic MR, Hutchison WD (2018) Neuronal inhibition and synaptic plasticity of basal ganglia neurons in Parkinson's disease. Brain 141:177-190.

Mirzaei A, Kumar A, Leventhal D, Mallet N, Aertsen A, Berke J, Schmidt R (2017) Sensorimotor processing in the basal ganglia leads to transient beta oscillations during behavior. J Neurosci 37:11220-11232.

Nambu A, Tokuno H, Takada M (2002) Functional significance of the cortico-subthalamo-pallidal 'hyperdirect' pathway. Neurosci Res 43:111117.

Neumann WJ, Degen K, Schneider GH, Brücke C, Huebl J, Brown P, Kühn AA (2016) Subthalamic synchronized oscillatory activity correlates with motor impairment in patients with Parkinson's disease. Mov Disord 31: $1748-1751$

Peng Y, Barreda Tomás FJ, Klisch C, Vida I, Geiger JR (2017) Layer-specific organization of local excitatory and inhibitory synaptic connectivity in the rat presubiculum. Cereb Cortex 27:2435-2452.

Ramirez-Zamora A, Giordano JJ, Gunduz A, Brown P, Sanchez JC, Foote KD, Almeida L, Starr PA, Bronte-Stewart HM, Hu W, McIntyre C, Goodman W, Kumsa D, Grill WM, Walker HC, Johnson MD, Vitek JL, Greene D, Rizzuto DS, Song D, et al. (2017) Evolving applications, technological challenges and future opportunities in neuromodulation: Proceedings of the Fifth Annual Deep Brain Stimulation Think Tank. Front Neurosci 11:734.

Reich MM, Steigerwald F, Sawalhe AD, Reese R, Gunalan K, Johannes S, Nick R, Matthies C, McIntyre CC, Volkmann J (2015) Short pulse width widens the therapeutic window of subthalamic neurostimulation. Ann Clin Transl Neurol 2:427-432.

Rosenbaum R, Zimnik A, Zheng F, Turner RS, Alzheimer C, Doiron B, Rubin JE (2014) Axonal and synaptic failure suppress the transfer of firing rate oscillations, synchrony and information during high frequency deep brain stimulation. Neurobiol Dis 62:86-99.

Sanders TH, Jaeger D (2016) Optogenetic stimulation of cortico-subthalamic projections is sufficient to ameliorate bradykinesia in 6-OHDA lesioned mice. Neurobiol Dis 95:225-237. 
Shen KZ, Johnson SW (2006) Subthalamic stimulation evokes complex EPSCs in the rat substantia nigra pars reticulata in vitro. J Physiol 573:697-709.

Smith Y, Bolam JP, Von Krosigk M (1990) Topographical and synaptic organization of the GABA-containing pallidosubthalamic projection in the rat. Eur J Neurosci 2:500-511.

Steiner LA, Neumann WJ, Staub-Bartelt F, Herz DM, Tan H, Pogosyan A, Kühn AA, Brown P (2017) Subthalamic beta dynamics mirror parkinsonian bradykinesia months after neurostimulator implantation. Mov Disord 32:1183-1190.

Stephenson-Jones M, Samuelsson E, Ericsson J, Robertson B, Grillner S (2011) Evolutionary conservation of the basal ganglia as a common vertebrate mechanism for action selection. Curr Biol 21:1081-1091.

Tinkhauser G, Pogosyan A, Tan H, Herz DM, Kühn AA, Brown P (2017a) Beta burst dynamics in Parkinson's disease OFF and ON dopaminergic medication. Brain 140:2968-2981.
Tinkhauser G, Pogosyan A, Little S, Beudel M, Herz DM, Tan H, Brown P (2017b) The modulatory effect of adaptive deep brain stimulation on beta bursts in Parkinson's disease. Brain 140:1053-1067.

Uematsu M, Hirai Y, Karube F, Ebihara S, Kato M, Abe K, Obata K, Yoshida S, Hirabayashi M, Yanagawa Y, Kawaguchi Y (2008) Quantitative chemical composition of cortical GABAergic neurons revealed in transgenic venus-expressing rats. Cereb Cortex 18:315-330.

Williams NR, Foote KD, Okun MS (2014) STN vs. GPi deep brain stimulation: translating the rematch into clinical practice. Mov Disord Clin Pract 1:24-35.

Wilson CL, Puntis M, Lacey MG (2004) Overwhelmingly asynchronous firing of rat subthalamic nucleus neurones in brain slices provides little evidence for intrinsic interconnectivity. Neuroscience 123:187-200.

Zheng F, Lammert K, Nixdorf-Bergweiler BE, Steigerwald F, Volkmann J, Alzheimer C (2011) Axonal failure during high-frequency stimulation of rat subthalamic nucleus. J Physiol 589:2781-2793. 